FZR-85

April 1995

Preprint

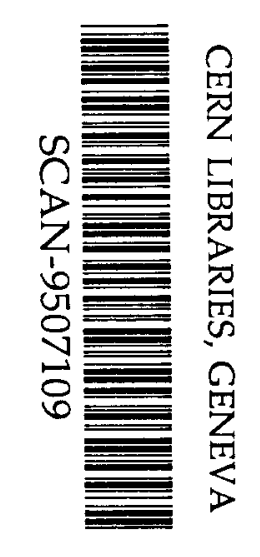

G. Pausch, M. Moszyński, D. Wolski,

W. Bohne, H. Grawe, D. Hilscher,

R. Schubart, G. de Angelis and M. de Poli

\title{
Application of the Pulse-Shape Technique to Proton-Alpha Discrimination in Si-Detector Arrays
}


Forschungszentrum Rossendorf e.V.

Postfach 510119 . D-01314 Dresden

Bundesrepublik Deutschland

Telefon (0351) 5912986

Telefax (0351) 5913700

E-Mail pausch@fz-rossendorf.de 
(Submitted to Nucl. Instr. and Meth. in Physics Research A)

\title{
Application of the Pulse-Shape Technique to Proton-Alpha Discrimination in Si-Detector Arrays
}

\author{
G. PAUSCH \\ Forschungszentrum Rossendorf e.V., Institut für Kern- und Hadronenphysik, \\ Postfach 5101 19, D-01314 Dresden, Germany \\ M. MOSZYŃski, D. Wolski \\ Soltan Institute for Nuclear Studies, Department of Nuclear Electronics, \\ PL-05-400 Otwock-Swierk, Poland \\ W. Bohne, H. Grawe, D. Hilscher \\ Hahn-Meitner-Institut Berlin GmbH, Bereich Festkörperphysik, \\ Glienicker Str. 100, D-14109 Berlin, Germany \\ R. SchuBART \\ II. Physikalisches Institut, Universität Göttingen, \\ Bunsenstraße 7-9, D-37073 Göttingen, Germany \\ G. DEANGELIS, M. DEPOLI \\ Laboratori Nazionali di Legnaro, INFN \\ Via Romea 4, I-35020 Legnaro (PD), Italy
}

The capability of the pulse-shape technique with reversed $n$-type Si detectors for discrimination of protons and alphas produced in fusion-evaporation reactions was tested at the VICKSI cyclotron in Berlin. We applied a zero-crossing technique which does not need any external time reference, and which can therefore be exploited at DC accelerators. Excellent proton-alpha discrimination in the full energy range of the evaporation spectra, but also charge and even isotope resolution for heavier ions produced in projectile fragmentation, was obtained with detectors of an existing Si ball. There is no doubt that the pulse-shape discrimination works well with detectors from serial production and under experimental conditions which are typical for nuclear structure studies. An application of this technique in Si detector arrays is obvious, but some special features must be considered in the design of the electronics. The particle discrimination depends strongly on the electric field distribution inside the detector. Stabilization of the bias voltage at the detector is therefore recommendet. A consequence of the rear-side injection mode is a strong variation of the charge-collection time with energy, charge, and mass number of the detected ion. To obtain a precise energy signal it is indispensable to correct for the ballistic deficit. 


\section{Introduction}

The European $\gamma$-spectrometer EUROBALL [1] is planned to be completed for first nuclear structure experiments in 1997. A combination of $4 \pi$ detectors for light charged particles (LCP) with this highly efficient $\gamma$-array opens a wide field of applications, such as exitchannel selection for exotic states and nuclei, charged-particle spectroscopy, and Doppler correction of $\gamma$-ray energies [2]. The physics case implies necessary attributes of such an ancillary detector:

1. Minimum interference with $\gamma$ detection - i.e. low mass (to minimize absorption and scattering of $\gamma$-rays), and a count-rate capability not limiting the data rate of the $\gamma$-array;

2. Maximum detection efficiency for LCP - i.e. a geometrical coverage close to $4 \pi$, and low thresholds for particle detection and identification;

3. Capability for energy spectroscopy of LCP - i.e. reasonable energy resolution, and a detector thickness which is sufficient to stop the particles of interest;

4. A very good identification of alpha and proton events within the whole range of the evaporation spectra.

Prototype LCP detectors, which have been used in conjunction with the first generation of $\gamma$-arrays - OSIRIS [3], NORDBALL [4], the $8 \pi$-spectrometer [5] - and more recently with EUROGAM [6] and GASP [7], approach these ambitions with different concepts, but each of them has its limitations: With LCP arrays based on CsI(Tl) scintillators and photodiode readout it is possible to achieve a high geometrical coverage [6] and low identification thresholds for protons and alpha particles [8]. On the other hand, the performance is limited by the large dead time associated with the long decay time of the light pulse, and by the relatively large absorption and scattering of $\gamma$-rays. In contrary, silicon balls with thin detectors exploiting the $\Delta \mathrm{E}$ technique for proton-alpha discrimination [4] are distinguished by small interference with $\gamma$ detection. However, the particle discrimination is rather poor at large angles with respect to the beam since the reaction kinematics reduces the laboratory energies of evaporated particles, which results in overlapping $\Delta \mathrm{E}$ spectra of alphas and protons. Another shortcoming of this concept, the impossibility to obtain energy spectra of LCP, can be overcome by adding thicker $E$ detectors as done in the ISIS $\Delta \mathrm{E}-\mathrm{E}$ ball [7]. In this case, the necessary support structure reduces the covered solid angle and increases $\gamma$-ray scattering and absorption.

The recent success in exploiting pulse-shape properties of reversed planar Si detectors for particle discrimination [9] suggests a new approach: By combining the design idea of the 162-element Si-ball commissioned at the Hahn-Meitner-Institut Berlin [10] with the pulse-shape discrimination technique and detectors of sufficient thickness it should be possible to construct a $\mathrm{Si}$ ball which meets nearly perfectly all requirements of an ancillary LCP detector for EUROBALL. However, before starting such a project it is necessary to study the performance of the pulse-shape method in more detail:

- What is the quality of particle separation for standard detectors of existing Si balls, with irregular shapes and irradiated edge regions, under realistic experimental conditions for nuclear structure experiments? 
- Which count rates can be tolerated?

- Is it possible to modify the zero-crossing technique applied in reference [9] in such a way that no external time reference is necessary? Detector arrays like EUROBALL are often operated at DC accelerators (tandems) where no time-of-flight start signal is available, which means that the reference signal must be derived from the particle detector itself (selftriggering).

- Is it possible to discriminate protons and alpha particles with efficiencies close to $100 \%$, i.e. in the full energy range of the evaporation spectra superimposed by the reaction kinematics?

- What are the spectroscopic properties of reversed Si detectors?

The aim of the present work was a detailed investigation of these questions. The study covered an optimization of the detector operation associated with the bias voltage. A zero-crossing technique with selftriggering of the detectors was successfully implemented, and results for LCP as well as for heavy-ion discrimination are presented.

\section{Experimental Details}

The experiment was performed at the VICKSI cyclotron of the Ion-Beam Laboratory (ISL), Hahn-Meitner Institute Berlin. Protons and alpha particles were produced in fusionevaporation reactions of $150 \mathrm{MeV}{ }^{36} \mathrm{Ar}$ projectiles hitting a $2 \mathrm{mg} / \mathrm{cm}^{2}$ nat $\mathrm{Ni}$ target. The beam intensity varied from 0.7 to $1.4 \mathrm{pnA}$, with typical values of $\approx 1 \mathrm{pnA}$. Two passivated ion-implanted Si detectors of irregular hexagonal shape (figure 1) were mounted at a distance of $15 \mathrm{~cm}$ from the target on movable arms, which allowed to change the angle $\theta_{l a b}$ with respect to the beam axis during the experiment. These detectors were taken from serial production of Eurisys Measurements for the Berlin Silicon Ball (BSB) [10], but in contrary to the BSB detectors the silicon structure was glued with the front contact (highfield side) onto the $630 \mu \mathrm{m}$ ceramics backing to enable reverse operation as proposed in [11]. The main characteristics of these detectors are summarized in table 1 . The distance from the target corresponds to a maximum opening angle of $\approx 14^{0}$. We did not use any aperture or absorber in front of the detectors, thus irradiating the whole detector faces including edge regions. To protect the detectors against $\delta$ electrons a high voltage of $+20 \mathrm{kV}$ was applied to the target. A spectroscopic ${ }^{239} \mathrm{Pu}^{241} \mathrm{Am}^{244} \mathrm{Cm} \alpha$ source mounted at the lower position of the target ladder allowed to perform energy calibration and resolution tests without opening of the reaction chamber. During beam experiments the detectors were shielded against alpha particles from the calibration source.

The block scheme of the electronics is shown in figure 2. We used a charge-sensitive preamplifier with a sensitivity of $\approx 2.2 \mathrm{mV} / \mathrm{MeV}(\mathrm{Si})$. The energy signal was shaped with a usual spectroscopy amplifier, the time constant varied from $0.5 \mu$ s to $2 \mu$ s (see discussion below). To get pulse-shape information we applied bipolar shaping and derived the zero-crossing time by means of a zero-crossing discriminator (ZCD) as described in ref. [9]. Following the ideas of refs. [11] and [9], a first time-to-amplitude converter (TAC1) measured the time difference $t_{Z C+T O F}$ between zero crossing and the succeeding RF signal of the cyclotron representing the time-of-flight (TOF) start reference. The 8:1 bunch 
suppression mode of VICKSI provided a bunch separation of 635 ns which was necessary to obtain a sufficient time range. This technique requires a pulsed beam or an adequate external TOF-start signal - which can, however, in case of $\gamma$-spectroscopy experiments in principle be delivered by the $\gamma$ detectors. To be independent of such an external reference we introduced a constant-fraction discriminator (CFD) which derived timing information from the leading edge of the (relatively slow) bipolar signal. This CFD was operated in rise-time compensating mode (internal delay $\approx 40 \mathrm{~ns}$ ) and started a second time-toamplitude converter (TAC2). By stopping TAC2 with the zero-crossing signal ( $\mathrm{ZC})$ of the ZCD we obtained the parameter $t_{Z C}$ representing the time difference between leading edge and zero crossing of the bipolar signal. This technique is very similar to the wellknown pulse-shape discrimination scheme which has been used as a standard method for neutron- $\gamma$ discrimination with scintillation detectors (see e.g. [12] and reference [13], p. 314). All three parameters - the energy deposition $E$ in the detector, and the two time differences $t_{Z C+T O F}$ and $t_{Z C}$ - were digitized simultaneously by means of a 4 -fold CAMAC ADC (ORTEC AD413A) and stored eventwise on disk using the CAMAC-based data acquisition system HOOPSY [14] of the HMI Berlin. The internal level discriminator (LD) controlling the ZCD triggered the data acquisition.

The bipolar shaping indicated by the shaping amplifier in figure 2 was performed in two different ways: We started with a usual spectroscopy amplifier (Canberra 2020) exploiting the bipolar output and a shaping time $\tau_{b i}=0.25 \mu \mathrm{s}$ as described in ref. [9]. To get a faster response we replaced this amplifier in a second step by two subsequent timing-filter amplifiers (ORTEC 474 and 579) with identical time constants of $\tau_{\text {diff }}=100-200$ ns and $\tau_{\text {int }}=50 \mathrm{~ns}$. By means of a digitizing oscilloscope we were able to visualize the typical response to detector signals during irradiation (figure 3 ).

In order to test the performance of the standard BSB detectors and, in particular, of the more general pulse-shape discrimination technique without external TOF-start reference for heavy-ion identification we performed a second run with a $510 \mathrm{MeV}{ }^{36} \mathrm{Ar}$ beam using the equipment and electronics described above. A beam of typically 0.1 pnA ( $8: 1$ bunch suppression mode) to $0.5 \mathrm{pnA}$ (without bunch suppression) was focussed onto mixed targets consisting of $1 \mathrm{mg} / \mathrm{cm}^{2}$ foils of different elements like $\mathrm{C}, \mathrm{Al}, \mathrm{Ni}, \mathrm{Ag}$, or $\mathrm{Au}$. Due to projectile breakup and evaporation from highly excited nuclei following complete or incomplete mass and momentum transfer a broad spectrum of heavy ions was produced. With the detectors positioned at relatively small angles with respect to the beam, $\theta_{l a b}=20-25^{\circ}$, we were able to check charge and mass resolution up to Argon $(Z=18)$.

\section{Discussion of Results}

The most important result of our test is that both zero-crossing techniques - with external TOF-start reference as well as with selftriggering of the detectors - are very well suited for identification of light charged particles in conjunction with detectors from serial production for an existing $\mathrm{Si}$ ball, the BSB. In fact we got excellent proton-alpha discrimination in the full energy range of evaporation spectra when plotting the parameters $t_{Z C+T} O F$ or $t_{Z C}$ versus the energy $E$ deposited in the detector (figures $4-5$ and $6 \mathrm{a}$ ). Moreover, the test with a $510 \mathrm{MeV}{ }^{36} \mathrm{Ar}$ beam demonstrated that the more general method deriving $t_{Z C}$ only from the bipolar signal (selftriggering) is capable of discriminating heavy ions in a wide dynamic range (figure $6 \mathrm{~b}$ ). The charge and mass resolution is comparable with the results 
published in [9], but in contrary to that reference it was obtained with totally exposed detectors of irregular shape and by means of a technique which can be applied without any restriction to experiments at $\mathrm{DC}$ accelerators.

In the $t_{Z C+T O F^{-}} E$ correlations, which we measured with an external time reference, the contribution of the TOF differences to the separation of the particle branches is small compared to the contribution of the pulse shapes. This is due to the TOF base of only $15 \mathrm{~cm}$, which results e.g. in a TOF difference of $\approx 5 \mathrm{~ns}$ for $5 \mathrm{MeV}$ protons and alpha particles whereas the spacing of the corresponding branches in the measured scatterplots is of the order of $100 \mathrm{~ns}$ (see figure 4). For Si balls operated inside $4 \pi$ neutron-or $\gamma$-detector arrays the situation would be comparable, because of the small size of such a ball. In spite of the negligible contribution of the TOF differences to the particle separation, an external time reference may be useful to reduce the expense of electronics or to simplify the triggering scheme in case that a Si-detector array is operated as a slave of another detection system. The necessary time resolution for such a reference signal indicating start of an event can be estimated from the time spacing $\Delta t_{Z C}$ of particle branches in the $t_{Z C}-E$ representation. Figure 7 shows projections of measured two-dimensional $t_{Z C}-E$ spectra onto the calibrated $t_{Z C}$ axis which correspond to small energy bins. It is evident that a time resolution of $\approx 5$ ns is sufficient for proton-alpha discrimination. A Si ball designed for LCP detection in $\gamma$-arrays can therefore be operated with a timing signal derived from the Ge detectors or the BGO shields as reference and trigger signal. However, isotope resolution in the mass range of carbon ions requires a better resolution of $<2 \mathrm{~ns}$ for the external time reference.

To check the tolerable count rate we varied the detector position. A decrease of $\theta_{l a b}$ enhances the rate and - because of the reaction kinematics - the laboratory energy of elastically scattered projectiles and recoiling target nuclei. Figure 5 shows $t_{Z C+T O F-E}$ scatterplots measured for $\theta_{\text {lab }}=80^{\circ}, 70^{\circ}$, and $60^{\circ}$, at count rates (for signals above the trigger threshold) of $\approx 4,7$, and $10 \mathrm{kHz}$, respectively, which are dominated by these heavy recoils. The heavy fragments can be discriminated without any problem, and no loss of resolution is observed for the proton and alpha branches. Nevertheless one should not renounce the application of absorber foils. The tolerable total irradiation dose is limited (see e.g. [15]), and screening the detectors from heavy recoils should increase the life time considerably.

A further aspect of our test was the optimization of the detector bias. The discussion of the physics effects responsible for particle discrimination (see ref. [11]) suggests that the spacing $\Delta t_{Z C}(E)$ of neighbouring particle branches and consequently the attainable particle resolution must be very sensitive to the electric field distribution $F(x)$ inside the detector. (The parameter $x$ characterizes the penetration depth viewed from the rear contact of the detector, see figure 8.) In case of reversed n-type Si-detectors, the pulse-shape differences are - at least for particles with a small range compared to the detector thickness - dominated by the plasma-erosion time, $\tau_{p l}$. According to the model of England et al. [16], $\tau_{p l}$ scales with the square root of the linear charge-carrier density $n$ in the ionization track (which is proportional to the electronic stopping power), and with the reciprocal of the electric field strength $F$. For ions with neighbouring mass or charge numbers but equal energies, generating ionization tracks of different lengths $(\Delta R=$ $R_{1}-R_{2}$, where $R_{1}$ and $R_{2}$ denote the range of the lighter and the heavier ion, respectively) and with different charge-carrier densities $\left(\Delta n=n_{1}-n_{2}\right)$, the relative variation of $\tau_{p l}$ is 
therefore in a first approximation given by

$$
\frac{\Delta \tau_{p l}}{\tau_{p l}} \propto\left(\frac{\Delta n}{2 n}-\frac{\Delta F}{F}\right)
$$

where

$$
\Delta F=\Delta R \cdot \frac{d F(x)}{d x}
$$

characterizes the variation of the electric field due to the different penetration depths of these ions. The field gradient, $f=d F(x) / d x$, has a constant value given by the resistivity of the material. Nevertheless the field contribution to the relative variation of the plasmaerosion time, $(\Delta F / F)=\Delta R \cdot d F(x) /(F \cdot d x)$, can be influenced by the detector bias $U_{D}$ (figure 8): For $U_{D}=U_{\text {dep }}$ (where $U_{\text {dep }}$ means the bias voltage which is necessary just for total depletion) the field $F$ at a certain position $x$ is lower than in case of overbiasing, which means a maximum for $\Delta F / F$. A lower detector bias therefore causes not only a larger absolute spacing of particle branches due to the reduced electric field, but also a larger relative variation of $\tau_{p l}$ for neighbouring ions. Due to this argument one expects best particle separation for a detector bias close to the depletion voltage. Such a behaviour is clearly observed in the experimental spectra (figure 4).

Another point demonstrated in figure 4 is the high sensitivity of the $t_{Z C+T O F^{-}} E$ correlation to the applied bias voltage. It is evident that small drifts of $U_{D}$, even of the order of a few volts, may disturb the particle separation tremendously. Such drifts are due to the temperature and count-rate dependence of the leakage current $I_{D}$, leading to a variation of the voltage drop at the filter resistor of the preamplifier. In the experiment we observed long-term variations of $I_{D}$ up to $150 \mathrm{nA}$ (see table 1). For a filter resistance of $100 \mathrm{M} \Omega$ - which is a typical value for charge-sensitive preamplifiers - this would result in an excessively large long-term drift of $U_{D}$ up to $15 \mathrm{~V}$. For practical applications these drifts must be kept small, either by choosing preamplifiers with a small filter resistance, or by stabilizing the bias voltage at the detector.

The strong variation of the charge-collection time with charge, mass, and energy of the detected ion in case of reversed n-type Si-detectors, still accentuated by a low bias, is advantageous for optimum particle discrimination. On the other hand, this variation complicates a correct energy measurement since the ballistic deficit is no more negligible and, moreover, dependent on all these parameters as well. This fact is reflected in the calibration spectra measured with a shaping-time constant of $\tau_{E}=0.5 \mu$ s for the energy signal (figure 9). A reduction of the detector bias to values in the range of the depletion voltage shifts the alpha peaks to lower channel numbers, as a result of the increasing charge-collection time which causes a considerable ballistic deficit. By means of a longer shaping time, $\tau_{E}=2.0 \mu \mathrm{s}$, the shift can be reduced (figure $9 \mathrm{~d}$ ). In conjunction with the strong energy dependence of the charge-collection time (see figure 4), the ballistic deficit results in a non-linear, particle-dependent energy calibration. This is illustrated in figure 10b. The displayed pulse-height spectra of alpha particles produced in fusionevaporation reactions, measured without any change of the experimental conditions except for the detector bias, were obtained as follows: We defined identification gates for alphas and protons, and projected these regions of the $t_{Z C}-E$ representation onto the $E$ axis. The alpha spectra were then normalized to the high-energy part of the corresponding proton spectra, assuming that the charge-collection time for signals of these protons is small enough to neglect the ballistic deficit. This normalization procedure is confirmed by the 
fact that the variation of the bias has no noticable effect on the shape of the proton spectra (figure 10a). However, the alpha spectra measured at lower values of $U_{D}$ are substantially distorted: Due to the increasing rise time of the charge signals with decreasing alpha energy, the ballistic deficit becomes more and more important, and shifts the pulse height of the energy signals $E$ to lower amplitudes. In spite of this effect the total number of registered alpha particles is only slightly reduced. A quantitative analysis, based on the discussed normalization to the proton spectra, results in a counting efficiency of $96.7 \%$ at $U_{D}=80 \mathrm{~V}$, if one assumes $100 \%$ at $U_{D}=100 \mathrm{~V}$ (figure $10 \mathrm{~b}$, inserted text). The pulse-shape discrimination method as described here is therefore ideal for LCP counting, e.g. for application in a $4 \pi \mathrm{Si}$-ball for exit-channel selection in nuclear structure physics. A detection and discrimination efficiency close to $100 \%$ for protons and alpha particles, independently of the reaction kinematics, is achievable without any doubt. However, charged-particle spectroscopy requires ballistic deficit correction (BDC). Corresponding techniques are well known from $\gamma$-spectroscopy with large Ge detectors and extensively discussed in reference [17].

\section{Conclusions}

Our experimental results demonstrate that the concept of a $\mathrm{Si}$ ball with reversed planar n-type detectors, exploiting the pulse-shape technique for particle discrimination, is practicable. The succesful implementation of selftriggering makes this method independent of any external time reference, which is very important e.g. for applications at DC accelerators. Using standard detectors of an existing Si ball in an experimental environment typical for nuclear spectroscopy we obtained excellent proton-alpha discrimination in the full energy range of evaporated particles. Count rates of $\approx 10 \mathrm{kHz}$ can be tolerated without loss of resolution. In addition, we demonstrated that the electronics scheme with selftriggering is capable of resolving elements and even isotopes of heavier ions in a wide dynamic range, with a resolution comparable to a previous experiment using an external time reference [9]. To obtain best particle resolution the detectors must be operated with a low bias voltage, preferably just with the depletion voltage. In this case the sensitivity of the charge-collection time to charge and mass, but also to the energy of the detected ion and to small variations of the bias voltage is at its maximum. An unavoidable consequence is a non-negligible ballistic deficit of the energy signal, depending on particle type and energy. For a precise energy measurement it is therefore indispensible to exploit spectroscopy amplifiers with ballistic deficit correction (BDC). Furthermore one has to take care that the variation of the bias voltage at the detector, e.g. due to a rising leakage current after irradiation causing an increased voltage drop in the preamplifier, is kept small.

Based on these results it is possible to design a $4 \pi$ Si-ball as an ancillary charged-particle detector for EUROBALL. Further applications of the investigated pulse-shape technique are obvious, e.g. in the field of heavy-ion physics where $4 \pi$ detectors with low identification thresholds for intermediate-mass fragments are required to study the properties of hot nuclear matter. 


\section{References}

[1] J. Gerl and R.M. Lieder (eds.), EUROBALL III - European $\gamma$-Ray Facility, GSI Darmstadt, Dec. 1992.

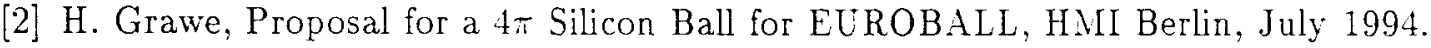

[3] D. Alber et al., Annual Report 1986, HMI-B 441 (1987) 127.

[4] T. Kuroyanagi et al., Nucl. Instr. and Meth. A316 (1992) 289.

[5] A. Galindo-Uribarri, Prog. Part. Nucl. Phys., Vol. 28 (1992) 463.

[6] J.N. Scheurer et al., Proc. Intern. Conf. on New Nuclear Physics with Advanced Techniques, Ierapetra, Greece, June 1991 (World Scientific 1992), p. 108.

[7] G. DeAngelis et al., LNL-INFN Report 72/93 (1993) 171.

[8] M. Moszyński, D. Wolski, T. Ludziejewski, S.E. Arnell, Ö. Skeppstedt, and W. Klamra, Nucl. Instr. and Meth. A336 (1993) 587.

[9] G. Pausch, W. Bohne, D. Hilscher, H.-G. Ortlepp, and D. Polster, Nucl. Instr. and Meth. A349 (1994) 281.

[10] W. Bohne, H. Morgenstern, and P. Figuera, HMI-B 497 (1992) 92, HMI-B 507 (1993) 79.

[11] G. Pausch, W. Bohne, and D. Hilscher, Nucl. Instr. and Meth. A337 (1994) 573.

[12] T.K. Alexander, and F.S. Goulding, Nucl. Instr. and Meth. 13 (1961) 244.

[13] W.R. Leo, Techniques for Nuclear and Particle Physics Experiments, 2nd Edition, Springer-Verlag Berlin Heidelberg New York 1994.

[14] G. Röschert, HOOPSY VMS User's Guide, Hahn-Meitner-Institut Berlin, May 1992.

[15] N. Kato, Nucl. Instr. and Meth. B51 (1990) 425.

[16] J.B.A. England, G.M. Field, and T.R. Ophel, Nucl. Instr. and Meth. A280 (1989) 291.

[17] M. Moszyński, and G. Duchêne, Nucl. Instr. and Meth. A308 (1991) 557. 


\section{Tables}

\section{Table 1}

Main characteristics of the detectors used in this experiment

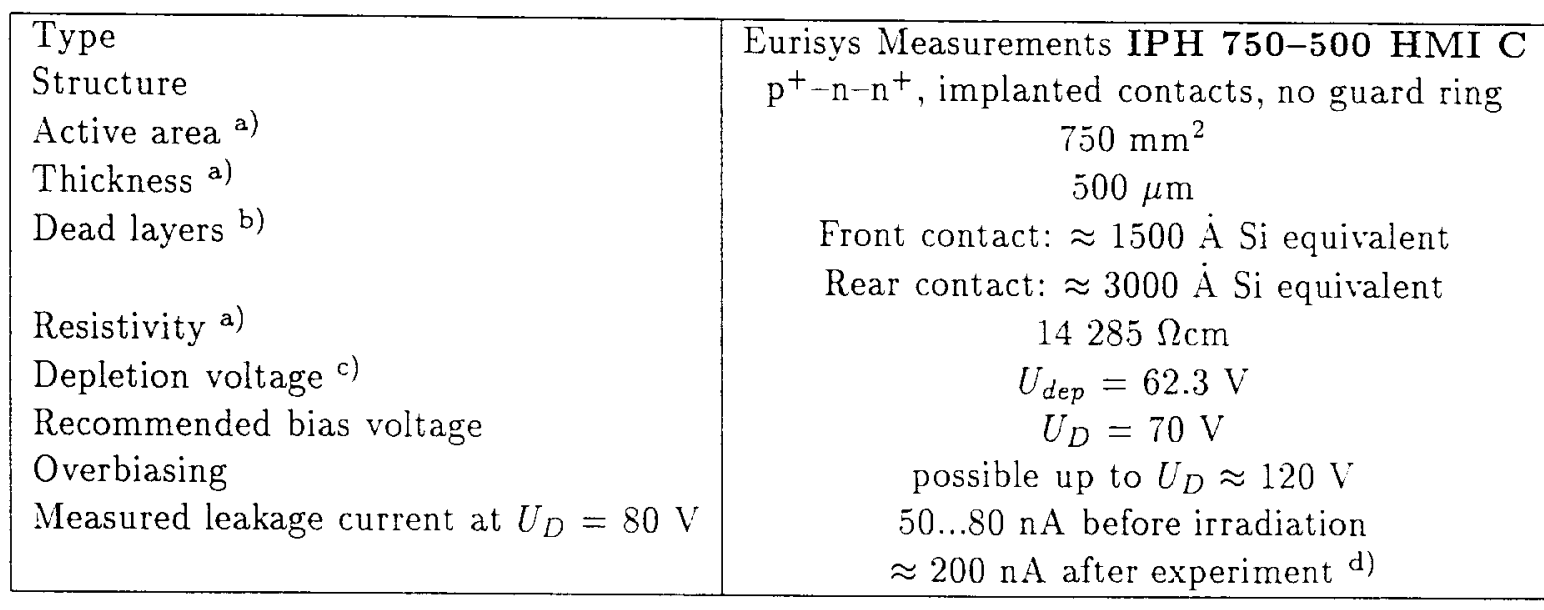

a) Values from data sheet

b) Information from Eurisys Measurements

c) Calculated from resistivity (see ref. [13], p. 226)

d) Estimated total dose of implanted ions: $\approx 2 \cdot 10^{8}$ particles (mainly $\mathrm{p}, \alpha$, and ${ }^{36} \mathrm{Ar}$ ) 


\section{Figure Captions}

Figure 1

View of the detectors used in this experiment (see table 1). These detectors are taken from serial production for the Berlin Silicon Ball [10], but glued with the front contact ( $p$ side) onto the ceramics backing which enables reverse operation.

\section{Figure 2}

Block scheme of the electronics used in our test: qPA - charge-sensitive preamplifier (IV69B, HMI Berlin), SPA - spectroscopy amplifier (Canberra 2022D), Shaping amplifier - see text, CFD - constant-fraction discriminator (ORTEC 935), ZCD - zero-crossing discriminator with level discriminator (LD) and zero-crossing $(\mathrm{ZC})$ outputs as described in ref. [9], TAC1, TAC2 - time-to-amplitude converters (ORTEC 566/567), DGG - delay and gate generator (ORTEC GG8010), RF - cyclotron RF signal (used as TOF-start reference).

*) Inspection point (see figure 3).

\section{Figure 3}

Typical bipolar signals at the inspection point (see figure 2) measured with a digitizing oscilloscope:

(a) $\mathrm{p}$ and $\alpha$ signals from $150 \mathrm{MeV}{ }^{36} \mathrm{Ar}+\mathrm{Ni}$, detector at $\theta_{l a b}=110^{\circ}$;

(b) $\mathrm{p}, \alpha$, and heavy-ion signals from $510 \mathrm{MeV}{ }^{36} \mathrm{Ar}+[\mathrm{Ag}+\mathrm{Au}]$, detector at $\theta_{\text {lab }}=25^{0}$.

The bipolar shaping is performed with two successive timing-filter amplifiers, ORTEC $474 / 579$, both operated with time constants of $\tau_{\text {diff }}=100 \mathrm{~ns}$ and $\tau_{\text {int }}=50 \mathrm{~ns}$.

\section{Figure 4}

Zero-crossing technique with external time reference, applied to proton-alpha discrimination: The $t_{Z C+T O F} E$ correlation, measured in the reaction $150 \mathrm{MeV}{ }^{36} \mathrm{Ar}+\mathrm{Ni}$ with a detector at $\theta_{l a b}=110^{\circ}$ (count rate $\approx 1 \mathrm{kHz}$ ), allows excellent $\mathrm{p}-\alpha$ discrimination. A variation of the detector bias from $75 \mathrm{~V}$ (a) to $100 \mathrm{~V}$ (d) demonstrates that best resolution is obtained with a low bias (see text). The discontinuity of the alpha and proton branches in the scatterplots is due to the limited time range for $t Z C+T O F$, given by the repetition rate of the beam bunchs.

\section{Figure 5}

Test of the count-rate dependence and the influence of scattered projectiles or recoiled target nuclei on the particle discrimination: The scatterplots were measured in the reaction $150 \mathrm{MeV}{ }^{36} \mathrm{Ar}+\mathrm{Ni}$, with the detector

(a) at $\theta_{l a b}=80^{\circ}$ (count rate $\approx 4 \mathrm{kHz}$ ),

(b) at $\theta_{\text {lab }}=70^{\circ}$ (count rate $\approx 7 \mathrm{kHz}$ ),

(c) at $\theta_{\text {lab }}=60^{\circ}$ (count rate $\approx 10 \mathrm{kHz}$ ),

and with a bias voltage of $U_{D}=100 \mathrm{~V}$. Beam current $(\approx 1 \mathrm{pnA})$ and target thickness $\left(\approx 2 \mathrm{mg} / \mathrm{cm}^{2}\right)$ are typical for nuclear-structure experiments. 


\section{Figure 6}

Test of the zero-crossing technique without external time reference for $\mathrm{p}-\alpha$ and heavy-ion discrimination: The $t_{Z C}-E$ plots were measured

(a) in the reaction $150 \mathrm{MeV}^{36} \mathrm{Ar}+\mathrm{Ni}, \theta_{l a b}=110^{\circ}, U_{D}=80 \mathrm{~V}$, trigger rate $\approx 1 \mathrm{kHz}$

(b) in the reaction $510 \mathrm{MeV}{ }^{36} \mathrm{Ar}+[\mathrm{Ni}+\mathrm{Al}+\mathrm{C}], \theta_{l a b}=25^{\circ}, U_{D}=80 \mathrm{~V}$, trigger rate $\approx 0.5 \mathrm{kHz}$. Note the isotope resolution in the element branches.

The $E$ scales were calibrated assuming a linear dependence of the pulse height on the energy deposition.

\section{Figure 7}

Projection of small $E$ bins of figure 6 onto the calibrated $t_{Z C}$ scale:

(a) and (b) - projections of figure $6 \mathrm{a}, E=8.0-8.2 \mathrm{MeV}$,

(c) and (d) - projections of figure $6 \mathrm{~b}, E=156-158 \mathrm{MeV}$.

\section{Figure 8}

Definition of the $x$ axis and sketch of the electric field distribution in a reversed $n$-type $\mathrm{Si}$ detector: A homogeneous resistivity - resulting in a homogeneous space-charge density of the depleted silicon, and thus in a constant field gradient - is assumed. A maximum of $d F(x) /(F \cdot d x)$ is obtained if the detector bias $U_{D}$ equals the depletion voltage, $U_{d e p}$.

\section{Figure 9}

Calibration spectra of a mixed ${ }^{239} \mathrm{Pu}^{241} \mathrm{Am}-{ }^{244} \mathrm{Cm} \alpha$ source, measured for different bias voltages $U_{D}$ and with different shaping-time constants $\tau_{E}$ of the spectroscopy amplifier generating the $E$ signal (see figure 2). The peak shift is due to the ballistic deficit which increases strongly with decreasing bias voltage.

\section{Figure 10}

Pulse-height spectra of LCP produced in the reaction $150 \mathrm{MeV}{ }^{36} \mathrm{Ar}+\mathrm{Ni}$ at $\theta_{l a b}=110^{0}$, measured with a shaping-time constant $\tau_{E}=0.5 \mu$ for different bias voltages:

(a) Pulse-height spectra of protons,

(b) Pulse-height spectra of alpha particles.

The peak shifts and the shape variations of the alpha spectra result from the energy-and bias-dependent ballistic deficit. The counting efficiency $\epsilon$ is nearly constant. 



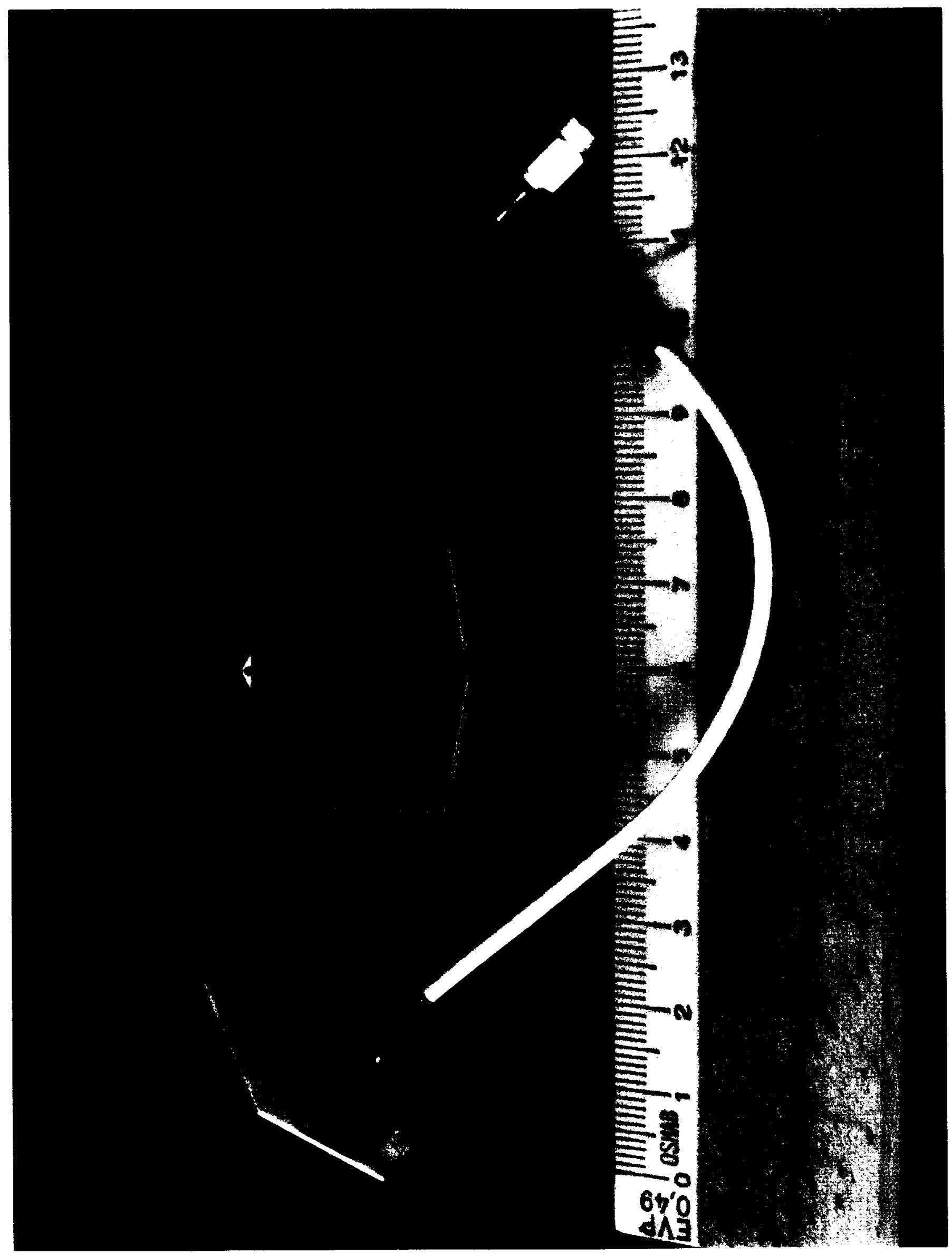

Figure 1 


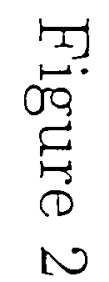

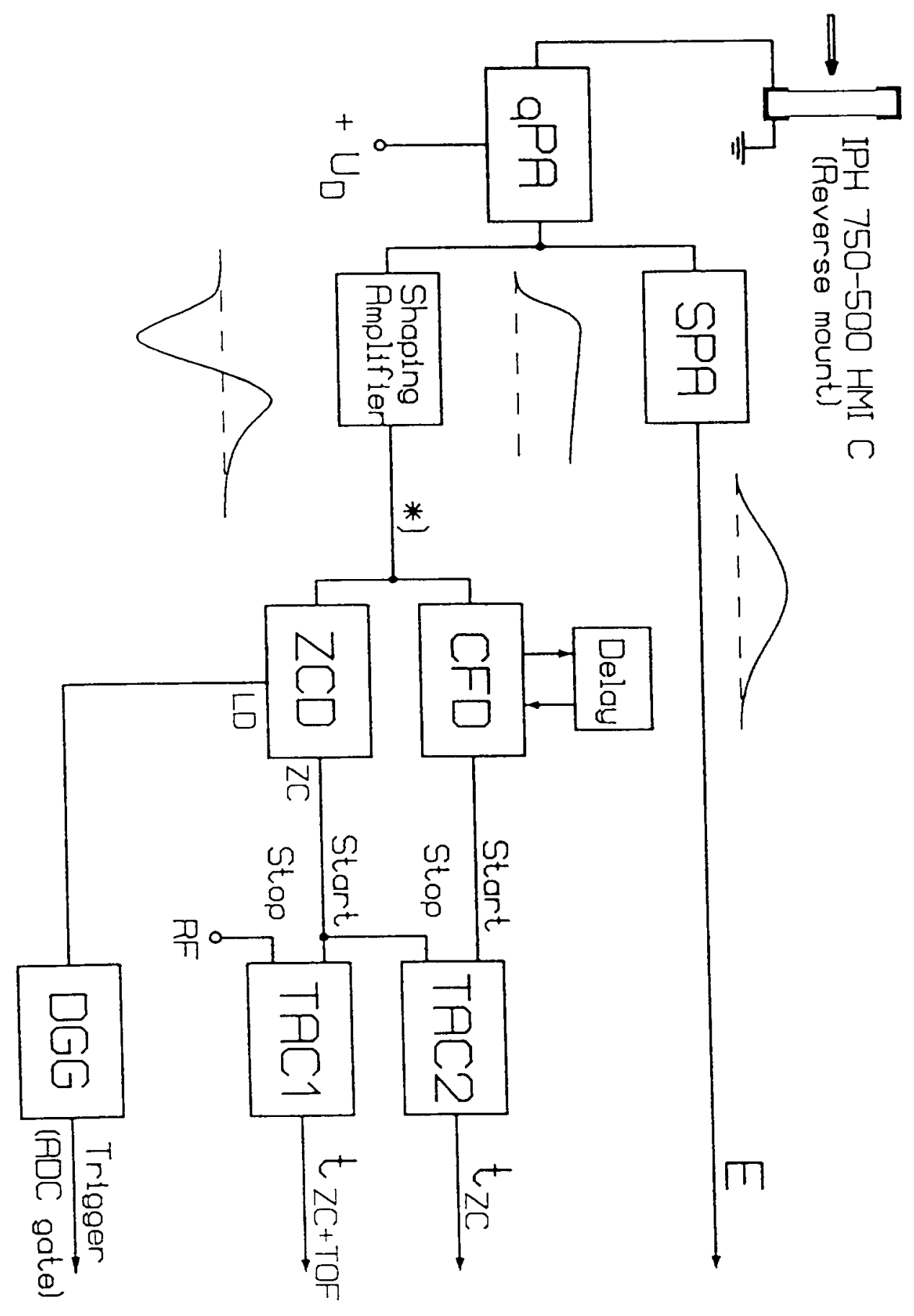



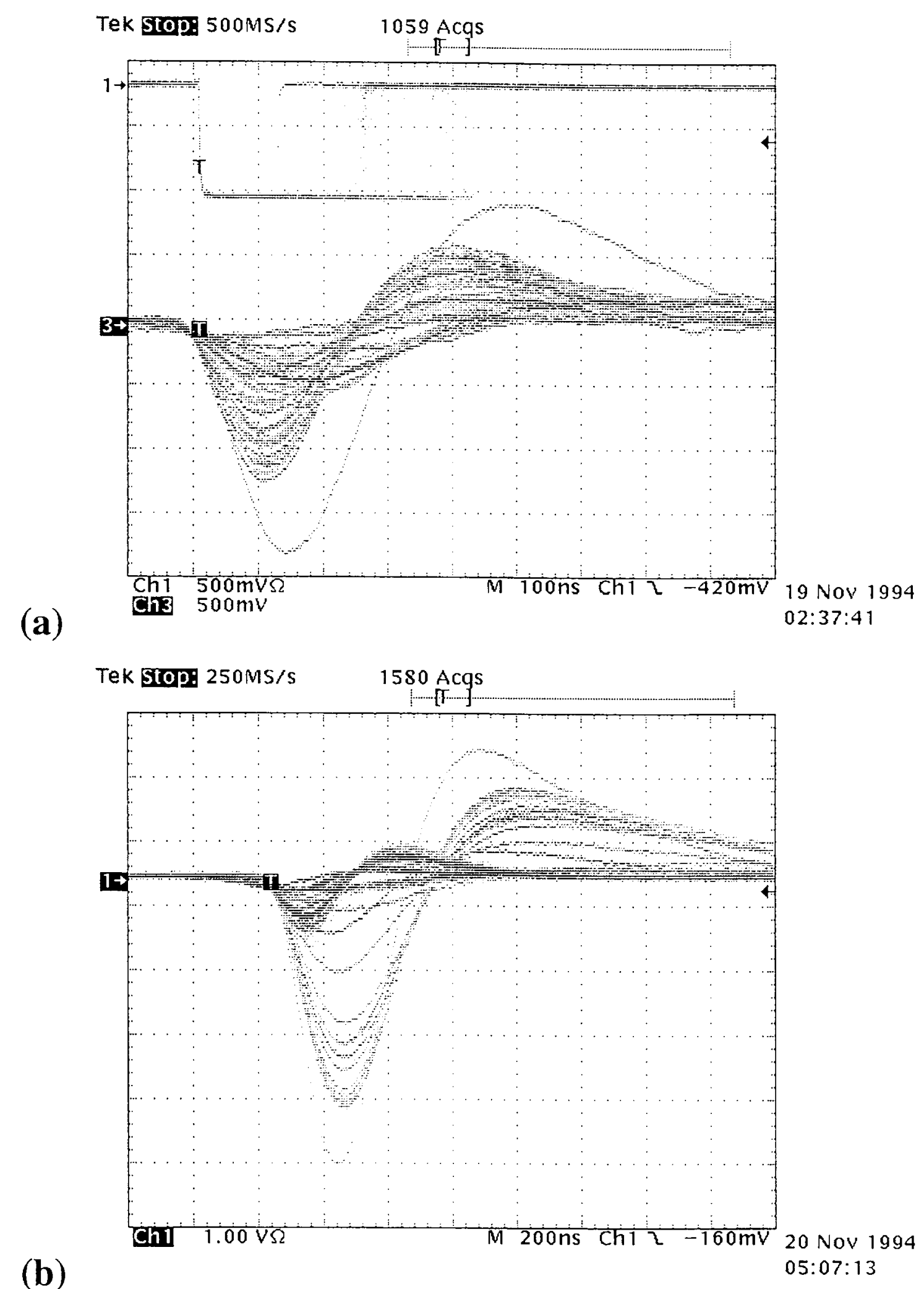

(b)

Figure 3 


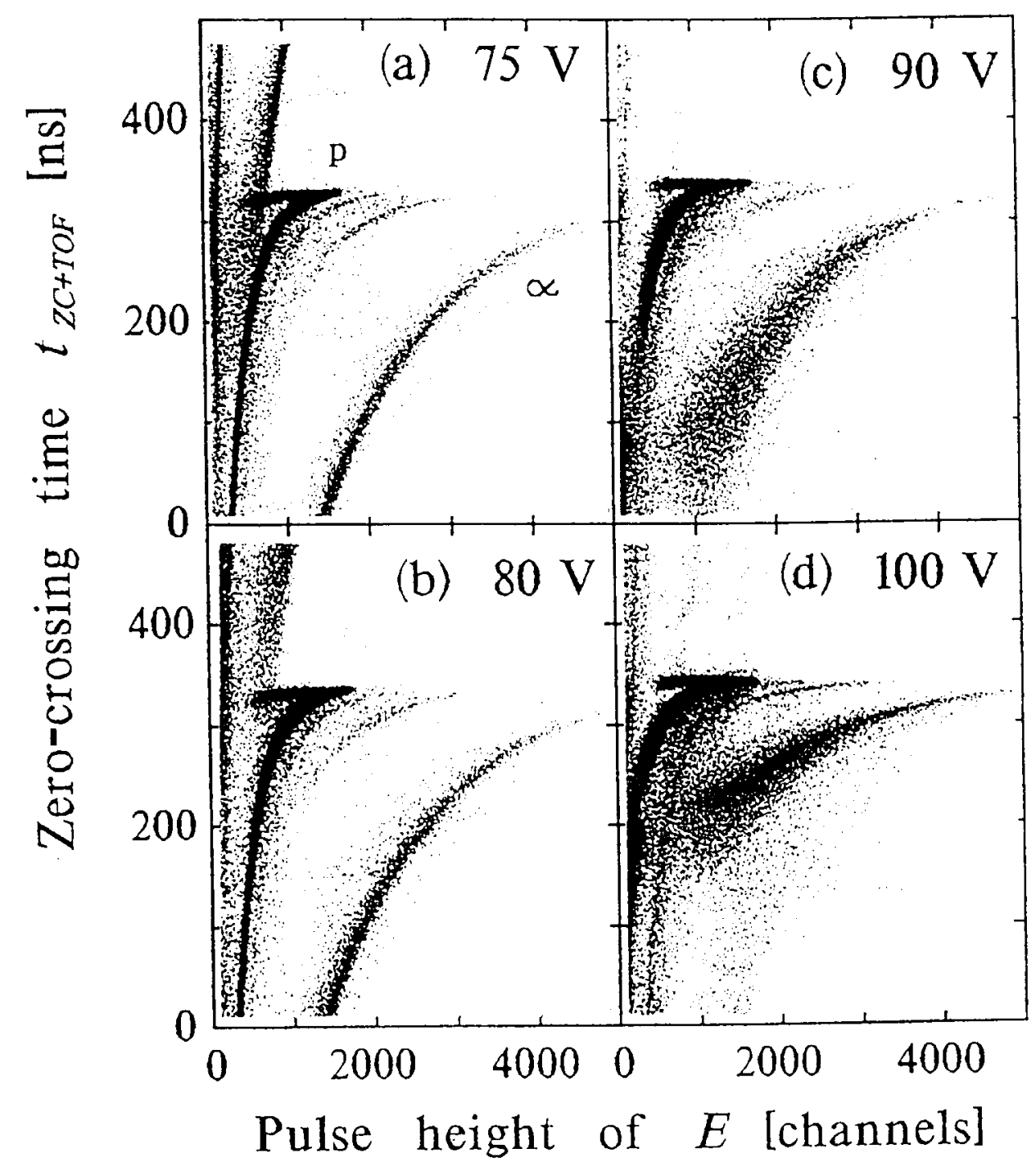

Figure 4 


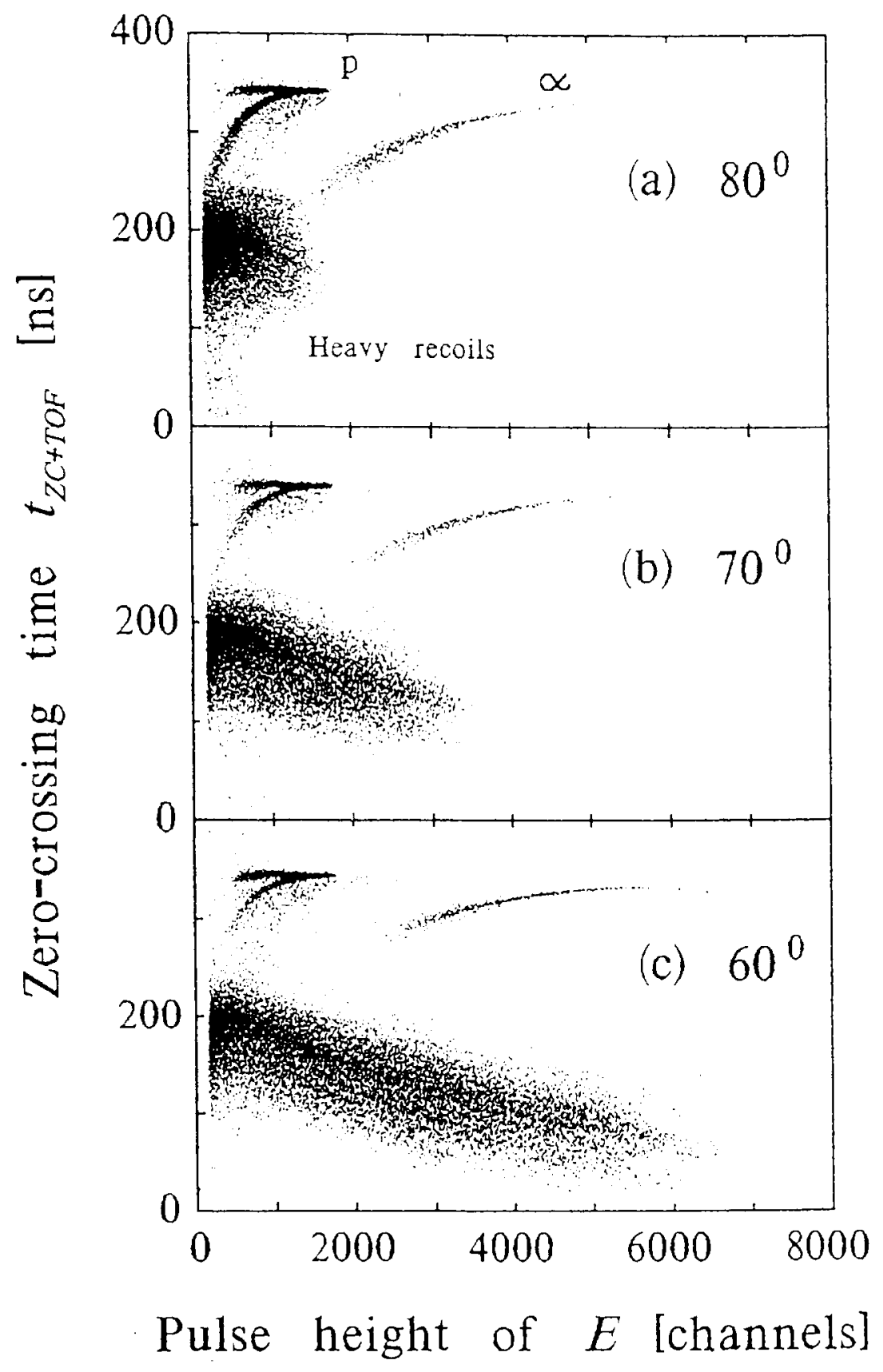

Figure 5 

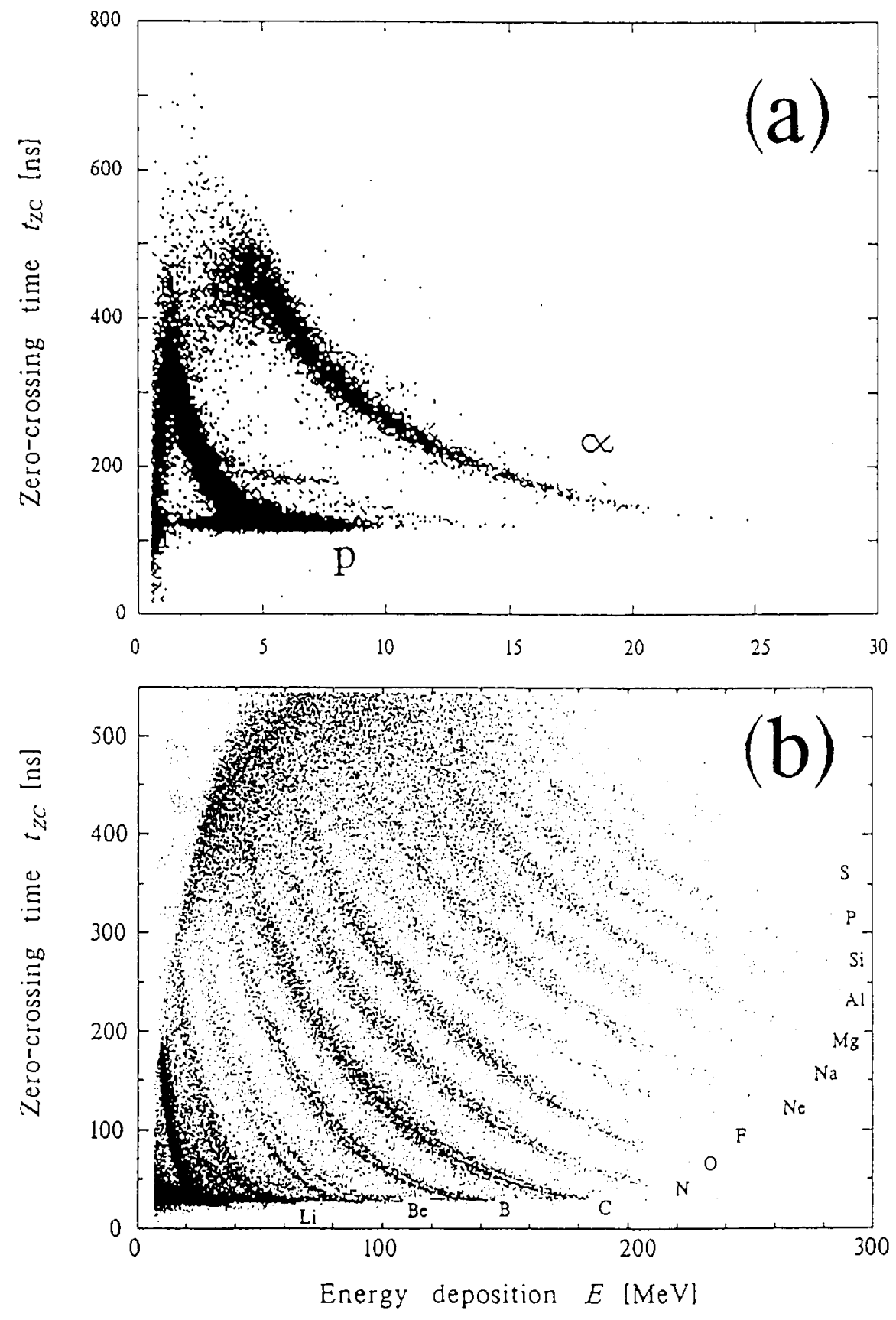

Figure 6 

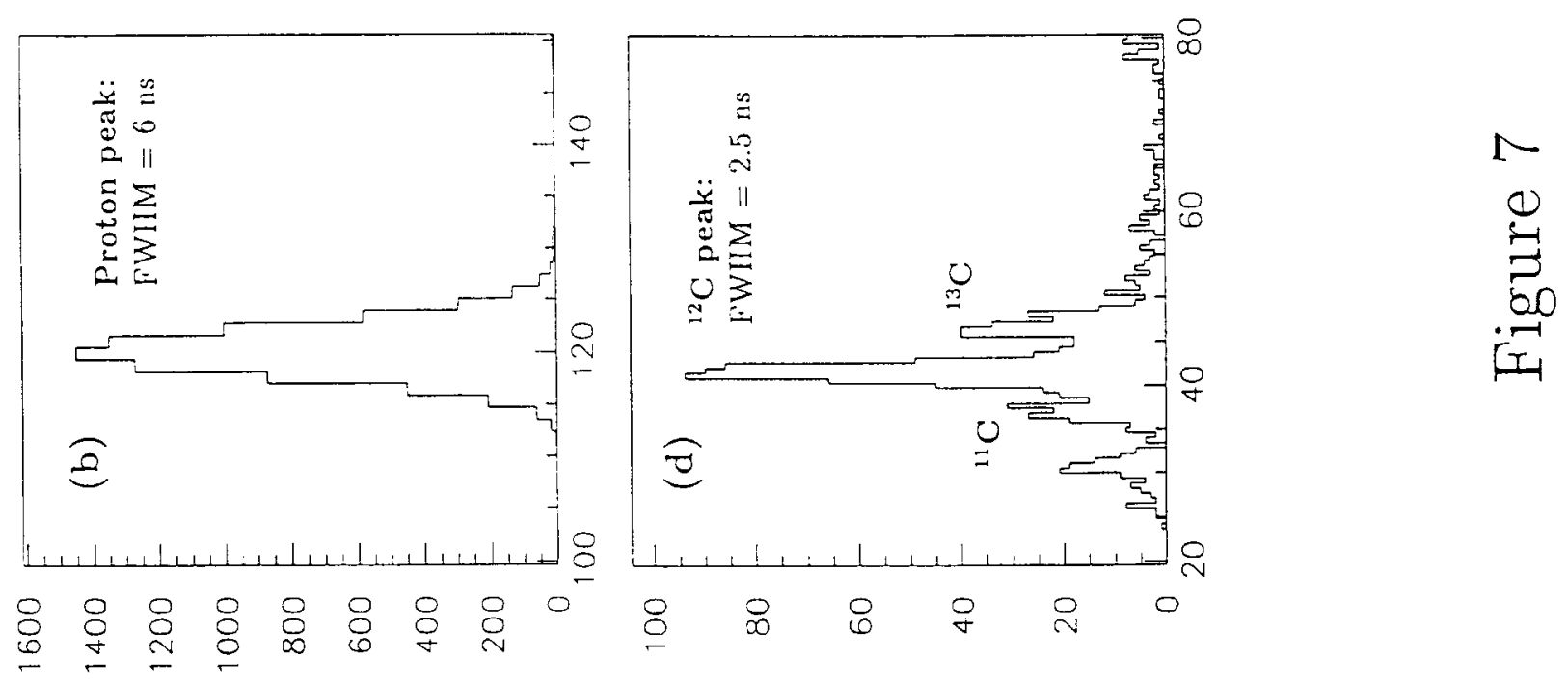

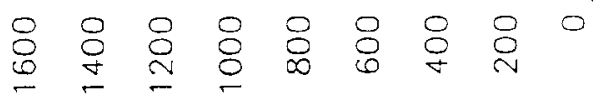
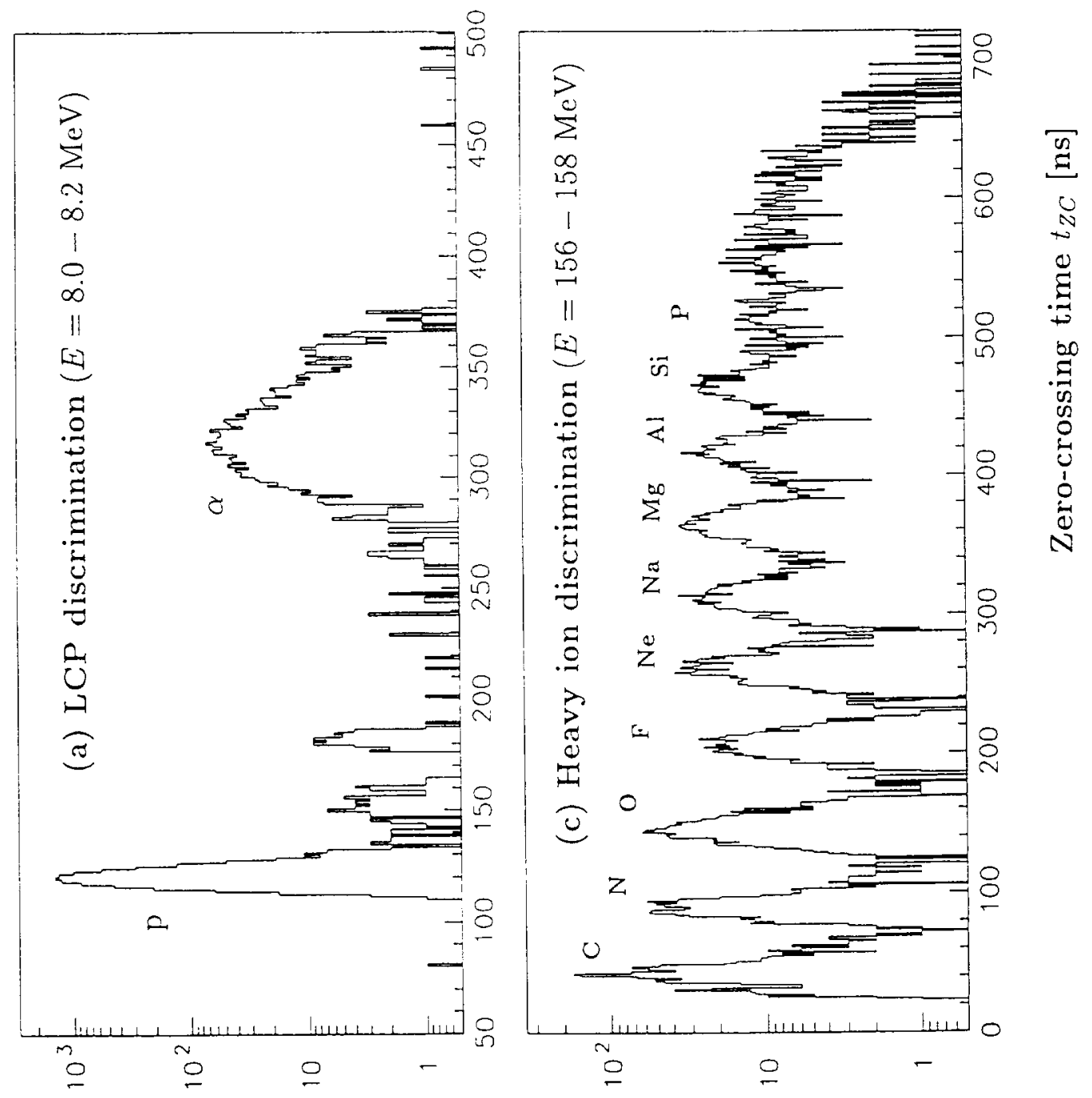


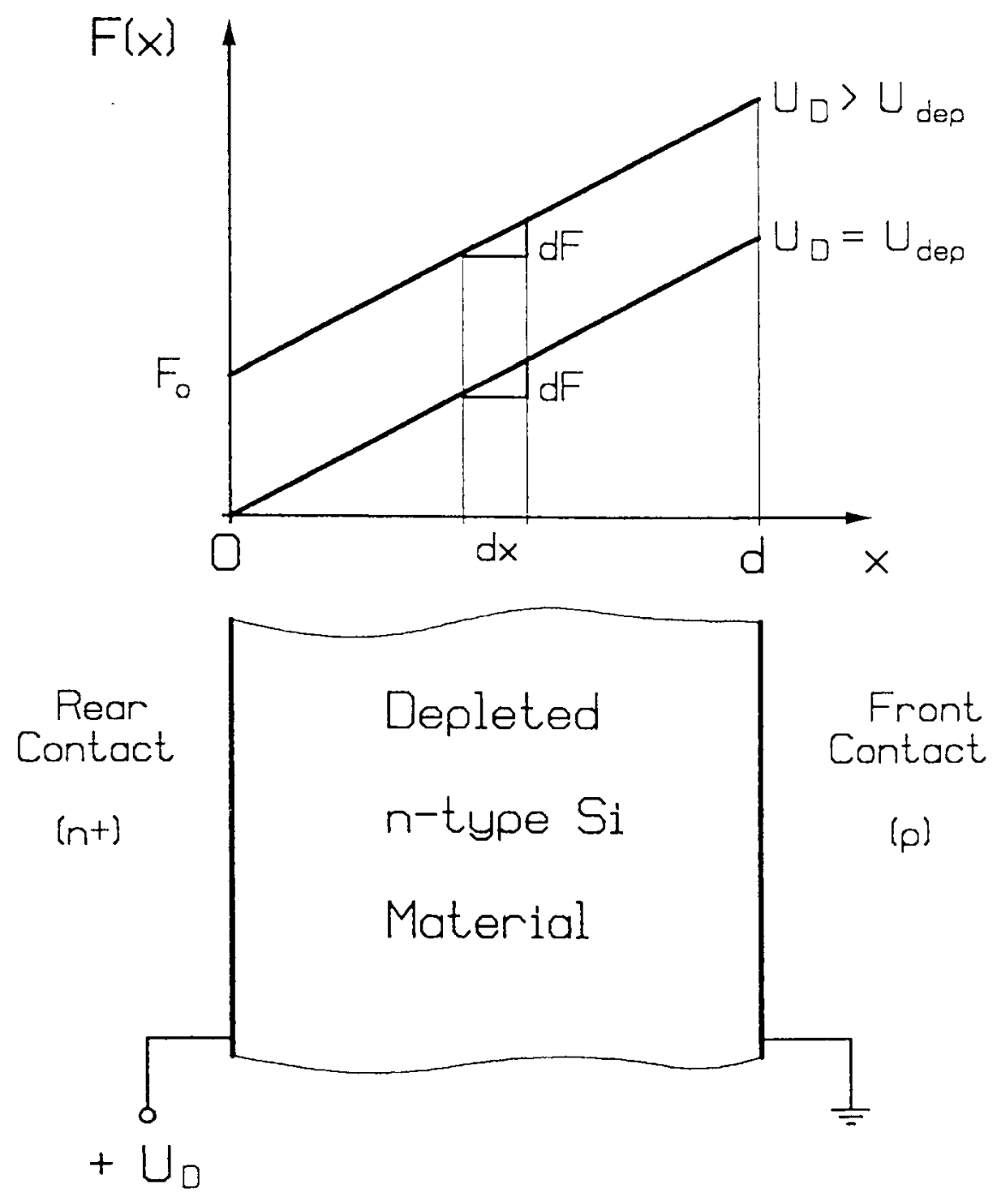

Figure 8 


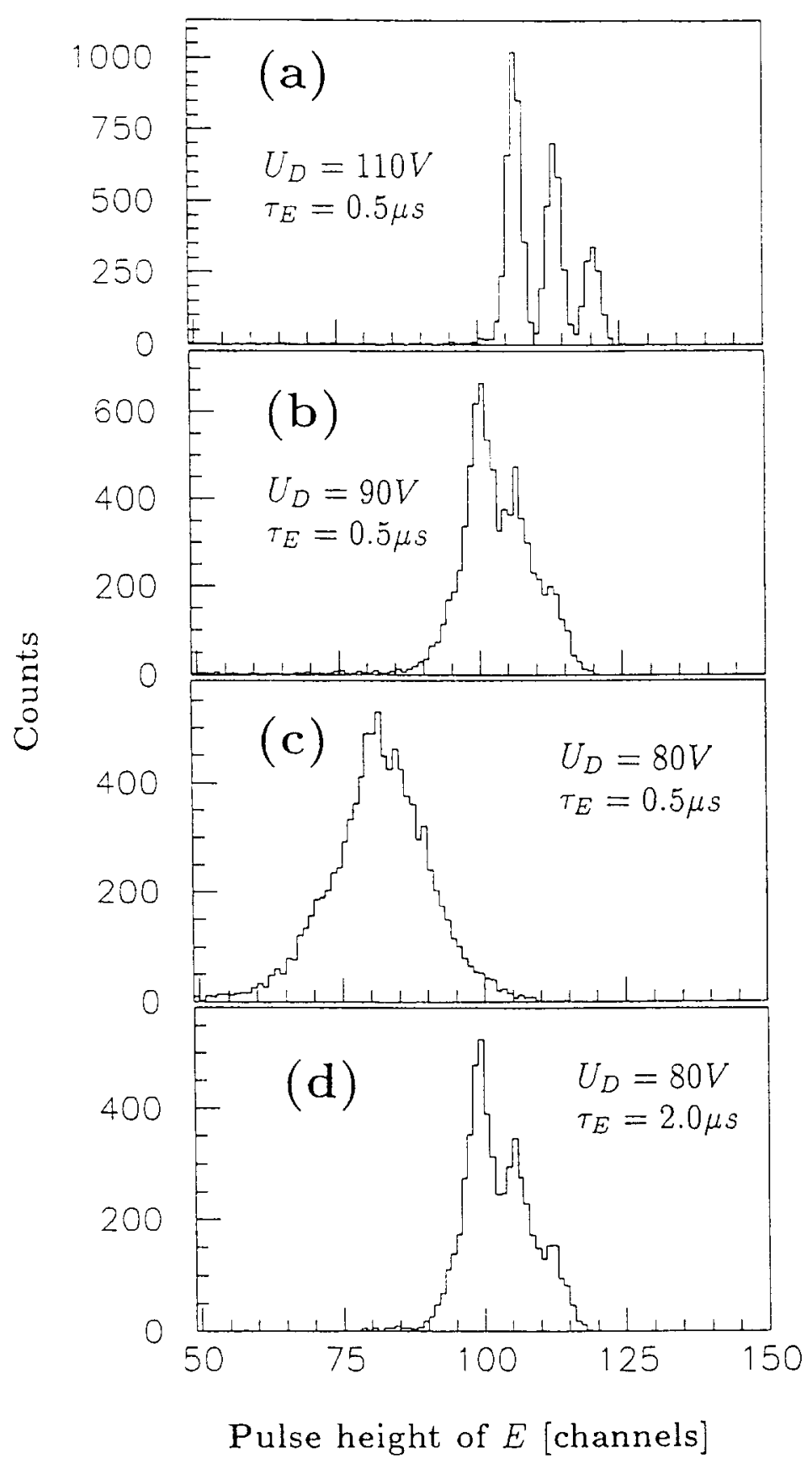

Figure 9 


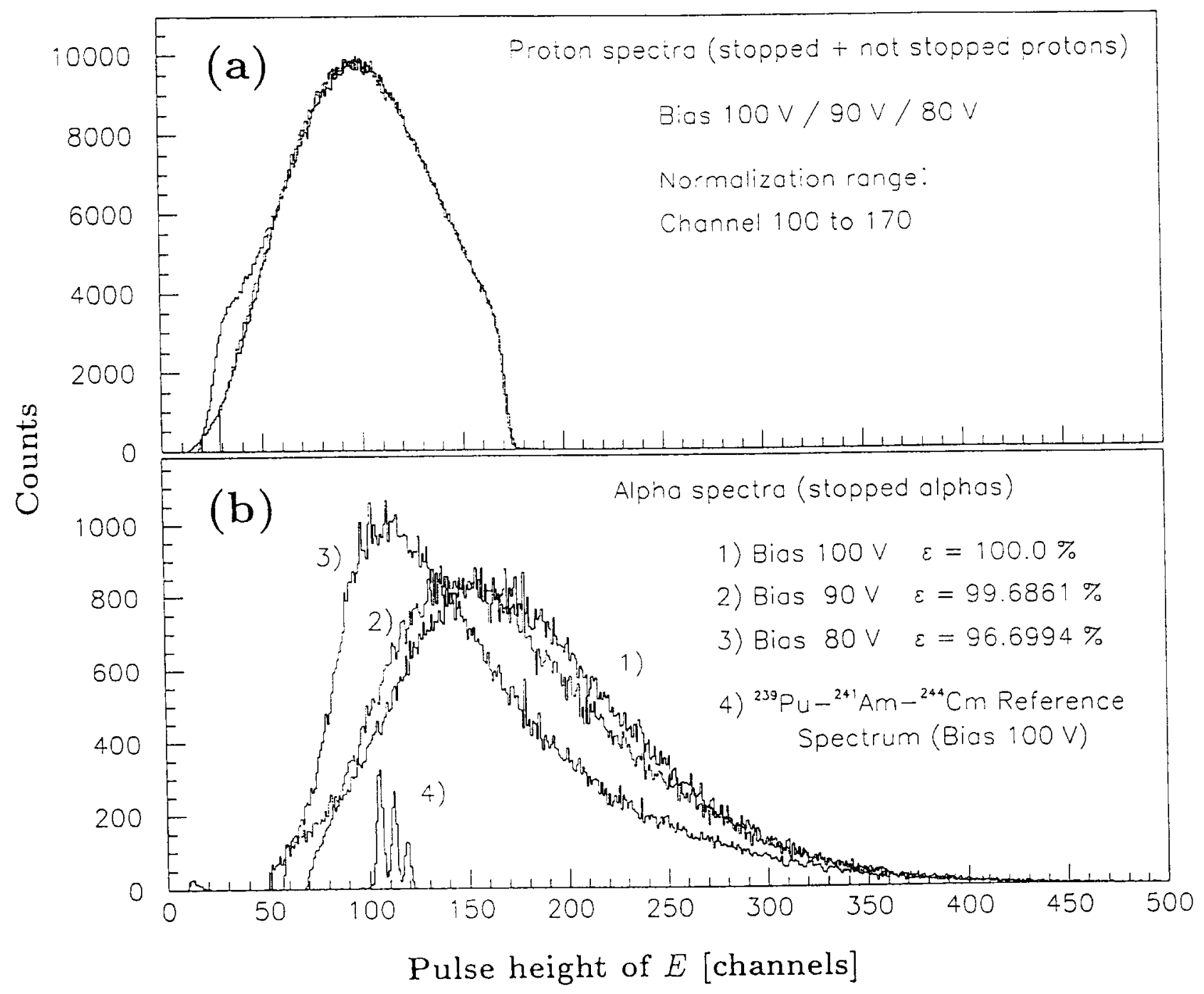

Figure 10 